\title{
The role of the F508C mutation in congenital bilateral absence of the vas deferens
}

\author{
Viktoria Havasi, $M D^{1}$, Steven Keiles, $M S^{2}$, Tina Hambuch, $P h D^{2}$, Eric J. Sorscher, $M D^{1}$, and Anja Kammesheidt, PhD ${ }^{2}$
}

\begin{abstract}
Purpose: Congenital bilateral absence of the vas deferens is a pathologic condition associated with normal spermatogenesis, azoospermia, and lack of both vasa deferentia. A significant association between mutations in the cystic fibrosis transmembrane conductance regulator gene among men with congenital bilateral absence of the vas deferens has been established. The objective of this study was to determine whether the $\mathrm{F} 508 \mathrm{C}$ variant in the cystic fibrosis transmembrane conductance regulator gene has a significant effect on congenital bilateral absence of the vas deferens prevalence, when present in conjunction with a second cystic fibrosis transmembrane conductance regulator disease causing mutation. Methods and Results: We compared the frequency of F508C in male subjects submitted for diagnostic testing on suspicion of cystic fibrosis or during cystic fibrosis carrier screening, to men with a clinical diagnosis of congenital bilateral absence of the vas deferens. Although frequencies of F508C did not vary significantly between 850 individuals undergoing cystic fibrosis carrier screening and those submitted for diagnostic testing on suspicion of cystic fibrosis, the frequency of $\mathrm{F} 508 \mathrm{C}$ in the congenital bilateral absence of the vas deferens population was significantly higher than expected $\left(\chi^{2}=6.95\right.$, corrected $\left.P=0.0486\right)$. Conclusion: We conclude that the F508C variant in cystic fibrosis transmembrane conductance regulator may represent a pathogenic defect and lead to congenital bilateral absence of the vas deferens when combined with a second cystic fibrosis transmembrane conductance regulator mutation. Genet Med 2008:10(12):910-914.
\end{abstract}

Key Words: cystic fibrosis, congenital bilateral absence of the vas deferens, F508C mutation

The etiology of congenital bilateral absence of the vas deferens (CBAVD) is not known, but a well-established connection exists between CBAVD and cystic fibrosis (CF). CF is characterized by recurrent pulmonary infections, elevated sweat chloride, pancreatic failure, hepatic insufficiency, and other glandular defects. ${ }^{1}$ Approximately $98 \%$ of CF males exhibit Wolffian duct abnormalities such as absence of the vas deferens, epididymis, seminal vesicles, or ejaculatory ducts. CF is caused by mutations in the cystic fibrosis transmembrane conductance regulator (CFTR) gene (OMIM 602421), which encodes an epithelial chloride channel.

More than 1,500 disease-associated CFTR mutations have been reported in the Cystic Fibrosis Mutation Database. ${ }^{2}$ These can be subdivided based on their molecular mechanism of action and include mutations that abrogate CFTR synthesis

\footnotetext{
From the ${ }^{1}$ Gregory Fleming James Cystic Fibrosis Research Center, University of Alabama at Birmingham, Alabama; and ${ }^{2}$ Ambry Genetics, 100 Columbia \#200, Aliso Viejo, California. Viktoria Havasi, MD, Gregory Fleming James Cystic Fibrosis Research Center, University of Alabama at Birmingham, 1918 University Boulevard, Birmingham, AL 35294. E-mail: vhavasi@uab.edu.

Disclosure: Steven Keiles, Anja Kammesheidt, and Tina Hambuch are employed by and hold stock in Ambry Genetics.

Submitted for publication April 4, 2008.

Accepted for publication September 16, 2008.

DOI: 10.1097/GIM.0b013e31818e594d
}

(e.g., premature stop codons), impair protein maturation, inhibit chloride conduction, or confer other abnormalities of CFTR cellular biology. ${ }^{3}$

Up to $99 \%$ of CBAVD patients carry at least one CF-associated CFTR mutation. ${ }^{4,5}$ Thus, CBAVD belongs to a group of CFTR related disorders and is considered an isolated, urogenital form of CF. ${ }^{6}$ The spectrum of the CFTR mutations in CBAVD, however, differs substantially from classical CF. Clinically "severe" CFTR mutations are typically found in CF individuals, whereas "mild" CFTR defects or compound heterozygous genotypes (a severe mutation together with a mild mutation or normal allele) are typically observed in CBAVD. ${ }^{7-9}$

The $\triangle$ F508 CFTR mutation (deletion of phenylalanine at amino acid position 508 within nucleotide-binding domain one) is the best described severe CFTR mutation. $\triangle$ F508 is often present in conjunction with a mild mutation (or no known second allelic defect) in CBAVD. Another CFTR mutation at the same residue-phenylalanine-to-cysteine replacement at CFTR position 508 - has also been described. This F508C mutation has traditionally been considered to represent a clinically silent polymorphism and not contribute to either CF or CBAVD.

The $\Delta$ F508 mutation decreases efficiency of protein folding and results in premature CFTR degradation in the endoplasmic reticulum, ${ }^{10}$ although $\mathrm{F} 508 \mathrm{C}$ is permissive for CFTR maturation. ${ }^{11}$ When Du et al. ${ }^{11}$ replaced F508 with cysteine in 
vitro, the protein matured, reached the cell surface and exhibited measurable chloride channel function. Cui et al. ${ }^{12}$ introduced cysteine at position 508 and conducted a more detailed study to examine modification of chloride channel activity. They demonstrated at the single-channel level that F508C increased channel mean closed time compared with wild-type CFTR, and concluded that the phenylalanine at position 508 may play an important role in channel gating.

The F508C mutation was first described in $1990,{ }^{13}$ and has been viewed primarily as a diagnostic confounder. Because F508C is located at the same amino acid position as $\Delta \mathrm{F} 508$, it may confer a nearly identical result in the setting of some heteroduplex CFTR genotyping methods, necessitating direct DNA sequencing to distinguish $\Delta \mathrm{F} 508$ from F508C. The Cystic Fibrosis Mutation Database ${ }^{2}$ and the NCBI SNP database ${ }^{14}$ do not relate F508C (rs 1800093) to any particular phenotype, or ascribe clinical significance to this polymorphism. Based on the critical location of F508C and earlier evidence of subtle defects in CFTR F508 $\mathrm{C} \mathrm{Cl}^{-}$channel activity, we investigated the incidence of F508C in patients with CBAVD compared with individuals screened either for CF carrier status or $\mathrm{CF}$, as a means of investigating whether any clinical significance might be associated with this variant.

\section{MATERIALS AND METHODS}

\section{Selection of study subjects}

Subjects in this study included men who had undergone full CFTR gene sequence analysis with the Ambry Test ${ }^{\mathrm{TM}}$ : CF at Ambry Genetics between January 2002 and June 2007. This group was comprised of 6,970 male patients, 850 of whom were analyzed for $\mathrm{CF}$ carrier screening purposes. All remaining specimens $(5,938)$ were submitted for diagnostic testing on suspicion of CF, or with a clinical diagnosis of CBAVD (182). The referral base for CBAVD typically includes male subjects with physical examination indicating absence of the vas deferens. The genetic test is sought as a means to determine associated CFTR mutation(s), or for assessment of CFTR status before in vitro fertilization. Individuals undergoing CF screening typically include subjects with a suspected CF phenotype (for whom genetic confirmation is sought), and those with an atypical presentation for whom a genetic test can establish the underlying diagnosis of CF.

\section{DNA isolation}

Genomic DNA (gDNA) was isolated from blood leukocytes according to standard procedures. gDNA was either isolated using the GFX genomic blood isolation kit (GE Healthcare, Chalfont St. Giles, UK), or using a Corbett XTractor automated system (Corbett Robotics, Sydney, Australia). DNA was assessed for quality and quantity by agarose gel electrophoresis.

\section{Gene analysis}

All subjects in this study were analyzed with the Ambry Test $^{\mathrm{TM}}$ : CF, which evaluates the CFTR gene by modified tem- poral temperature gradient electrophoresis analysis (mTTGE) followed by dye terminator DNA sequencing of suspect regions. The test covers all exons, plus at least 20 bases $5^{\prime}$ and $3^{\prime}$ into each intervening sequence, and select deep intronic mutations. Briefly, all exons and relevant intronic regions were amplified using polymerase chain reaction (PCR). Standard PCR amplification was performed using HotStarTaq Master Mix (Qiagen, Valencia, CA, USA) with 100-150 ng input gDNA per reaction. Typical PCR conditions were 1 cycle: $95^{\circ} \mathrm{C}$ for 15 minutes, 35 cycles: $94^{\circ} \mathrm{C}$ for 30 seconds, $\mathrm{X}^{\circ} \mathrm{C}$ for 30 seconds, $72^{\circ} \mathrm{C}$ for 30 seconds, 1 cycle: $72^{\circ} \mathrm{C}$ for 10 minutes (annealing temperature (X) was adjusted based on the particular primer pair). Before gel analysis, PCR products were denatured and slowly cooled to allow for maximal heteroduplex formation. For a subset of CFTR regions, DNA was mixed with known wild-type DNA samples to facilitate detection of homozygous mutations. PCR products were then processed for mTTGE on DCode gels (BioRad, Hercules, CA, USA) and polyacrylamide gels were analyzed for the presence of mutations after staining in ethidium-bromide and image capture under UV using a GelDoc system (BioRad, Hercules, CA, USA). Gel analysis was performed by two technologists and fragments scored against known controls. By this protocol and by incorporating overlapping mTTGE designs into the analysis, the method has been found to have comparable sensitivity and specificity to direct dye terminator sequencing.

Regions indicating the presence of a mutation by mTTGE were processed for dye terminator sequencing. Apparently affected exons were first amplified with a unique primer set using Taq PCR Master Mix (Qiagen, Valencia, CA, USA). Typical PCR conditions were 1 cycle: $95^{\circ} \mathrm{C}$ for 5 minutes, 35 cycles: $94^{\circ} \mathrm{C}$ for 30 seconds, $\mathrm{X}^{\circ} \mathrm{C}$ (see above) for 30 seconds, $72^{\circ} \mathrm{C}$ for 30 seconds, 1 cycle: $72^{\circ} \mathrm{C}$ for 10 minutes. PCR products were analyzed via agarose gel electrophoresis, followed by treatment with ExoSAP-It (USB, Cleveland, OH, USA) according to manufacturer's recommendations. Standard dye terminator cycle sequencing (Beckman Coulter, Fullerton, CA, USA) was conducted followed by analysis on a CEQ8000 capillary electrophoresis sequencer. Exons were sequenced in both sense and antisense directions to identify the precise nucleotide variation. All reported variations follow the nomenclature based on GenBank entry NM_000492 and the public database for CFTR. ${ }^{2}$

\section{Statistical analysis}

A 3-way $\chi^{2}$ contingency test was performed to ascertain the significance of F508C frequency differences among the following populations: (1) Patients submitted for diagnostic testing on suspicion of CF, (2) individuals screened for CF carrier status, and (3) individuals with a clinical diagnosis of CBAVD. The nature of the data collection and analysis preclude conclusions to be drawn regarding ethnic background, although no large differences in ethnic composition between the groups would be expected. Statistical analyses were performed using the VassarStats web tool ${ }^{15}$ which is operated by the Vassar College Statistics Department. Because F508C was present in fewer than five subjects in two of the three study groups, a Monte 
Carlo simulation of a three categorical sampling distribution was run for an additional 10,000 samples. Pairwise comparisons between the subdivided groups and odds ratios were also calculated by this program.

\section{RESULTS}

Genotype data for F508C was obtained from 850 subjects undergoing complete CFTR sequence analysis strictly for CF carrier screening and a group of 182 subjects with a clinical diagnosis of CBAVD. In addition, data from 5,938 patients

Table 1

Genotypes of CBAVD patients

\begin{tabular}{llclc}
\hline Patient & Mutation 1 & Mutation 2 & \multicolumn{1}{c}{ Clinical history } & Age \\
\hline 1 & DeltaF508 & F508C & CBAVD & 39 \\
2 & DeltaF508 & F508C & CBAVD, Sinusitis, Asthma & 34 \\
$3^{a}$ & L206W & F508C & CBAVD & 40 \\
\hline
\end{tabular}

${ }^{a}$ Family studies were not performed to determine if these variants are in position cis or trans.

Table 2

Genotypes of patients submitted for diagnostic testing on suspicion of CF

\begin{tabular}{llccc}
\hline Patient & Mutation 1 & Mutation 2 & Clinical history & Age \\
\hline 1 & G551D & F508C & Positive newborn screen & $\begin{array}{c}>1 \\
\text { mo }\end{array}$ \\
2 & V754M & F508C & Clinical suspicion of CF & $\begin{array}{c}1 \\
\text { mo }\end{array}$ \\
3 & DeltaF508 & F508C & Clinical suspicion of CF & $24 \mathrm{yrs}$ \\
4 & DeltaF508 & F508C & Clinical suspicion of CF & $11 \mathrm{yrs}$ \\
5 & DeltaF508 & F508C & Clinical suspicion of CF & $32 \mathrm{yrs}$ \\
\hline
\end{tabular}

CF, cystic fibrosis. who were submitted for diagnostic testing on suspicion of CF were analyzed.

Among the 850 individuals who were referred solely for CF carrier screening, $3(0.35 \%)$ carried the F508C variant without a second variant. As expected for a carrier screen, none carried F508C with a second variant. When CFTR from 182 individuals with a clinical diagnosis of CBAVD was analyzed, a total of three $(1.65 \%)$ individuals were identified to carry the F508C in addition to another mutation (Table 1). Comparatively, the allele frequency of F508C in the CBAVD population (3/364 alleles) was 4.68 times more common than in the CF carrier screening population ( $3 / 1700$ alleles), conferring an odds ratio of 4.70 (Table 3).

Further analysis was also conducted to determine the incidence of F508C among the 5,938 male individuals submitted for diagnostic testing on suspicion of CF. In 18 specimens submitted for diagnostic testing on suspicion of CF $(18 / 5,938=$ $0.303 \%)$, F508C was the only detected variant. In five individuals $(5 / 5,938=0.084 \%)$, the F508C variant was present along with a second mutation. (Table 2) Three subjects exhibited a S1251N-F508C genotype (with and without an additional third variant) and were excluded from the analysis because the CF associated variant S1251N is known to occur in cis with F508C. When comparing the allele frequencies of F508C in the group of patients with CBAVD (3/364 alleles) to those for whom F508C was observed with a second CFTR mutation (5/ 11876 alleles), F508C among CBAVD patients was 19.6 times more frequent.

Two-way $\chi^{2}$ tests revealed that the frequency of F508C was significantly higher in the CBAVD group than any other group (Table 3). Specifically, the frequency of the F508C allele in the CBAVD group was significantly increased compared with the entire group of patients submitted for diagnostic testing on suspicion of having CF (23/11876 alleles) $\chi^{2}=4.01(P=$ 0.0376 ) and when this group was subdivided to reflect those

Table 3

Frequencies of F508C among individuals referred for CF carrier screening $(\mathrm{n}=850)$, clinical diagnosis of CBAVD $(\mathrm{n}=182)$, and submitted for diagnostic testing on suspicion of CF $(n=5938)$

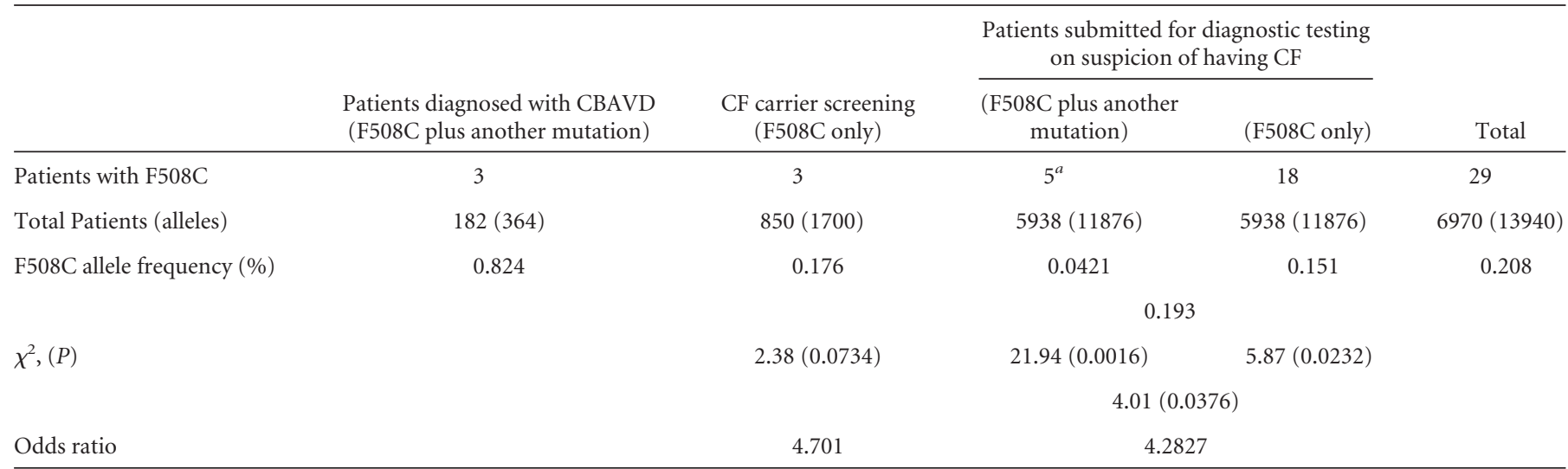

Chi square and odds ratios are shown for the group with a diagnosis of CBAVD compared with the CF carrier screening, patients submitted for diagnostic testing on suspicion of CF, or all other pooled groups in pairwise comparisons. $P$ values shown are corrected for 10000 sample simulation.

${ }^{a}$ Determined to carry one disease-associated CFTR mutation in addition to F508C.

$\mathrm{CF}$, cystic fibrosis; CBAVD, congenital bilateral absence of the vas deferens; CFTR, cystic fibrosis transmembrane conductance regulator. 
carrying a second known CF-causing mutation (5/11876 alleles) $\chi^{2}=21.94(P=0.0016)$ and those who only carried the F508C (18/11876 alleles) $\chi^{2}=5.87(P=0.0232)$. Direct comparison of the CBAVD group to the CF carrier screen group (3/1700 alleles) yielded a $\chi^{2}$ of 2.38 (corrected $P=0.0734$ ). This nominal $P$ value is a result of the low F508C frequency in the general population; a comparison of the $\mathrm{F} 508 \mathrm{C}$ frequencies between the CF carrier screen group and the group submitted for diagnostic testing on suspicion of CF showed no detectable difference $\left(\chi^{2}=0, P=1\right)$.

In a more robust three-way comparison, the frequency of F508C in individuals with CBAVD was significantly increased relative to both the CF carrier screen group (3/1700) and the group submitted for diagnostic testing on suspicion of CF (23/ 11876) $\left(\chi^{2}=6.95, P=0.031\right)$. The Monte Carlo simulation for 10,000 replications yielded a cumulative probability of 0.0486 .

\section{DISCUSSION}

The phenylalanine-cysteine change was originally observed in two healthy persons by Kobayashi et al., ${ }^{13}$ and has subsequently been reported in the literature in at least 12 other individuals. Four of these were described as normal, healthy subjects without any CF symptoms, ${ }^{16-19}$ five were CF patients ${ }^{20-22}$ and three were CBAVD subjects. ${ }^{23,24}$ Among the CF patients carrying F508C, other CFTR alterations (i.e., non F508C) have sometimes been invoked to explain a CF phenotype. Overall, F508C has been thought of as a benign variant.

In 1993 Meschede et al. ${ }^{23}$ first described the presence of F508C in a surgically documented case of CBAVD. Except for childhood pneumonia that individual had no typical CF symptoms and exhibited sweat electrolytes in the normal range. Later, Dork et al. ${ }^{24}$ examined a cohort of 106 German CBAVD subjects and identified F508C in three of them ${ }^{24}$ (including one of the three patients who had previously been described by Meschede et al.). F508C was not detected in 94 normal subjects or in $500 \mathrm{CF}$ subjects in the same study. ${ }^{24}$ These findings suggested that F508C might act as a CBAVD specific "mild" mutation with reduced penetrance, but that the pathogenic role of the defect would require further investigation.

In the present study, we examined the frequencies of F508C in a large group of DNA samples from a total population of 6,970 men, which on submission to our clinical laboratory had either been classified as possible $\mathrm{CF}$ carriers, clinical diagnosis of CBAVD or submitted for diagnostic testing on suspicion of CF. CBAVD is a rare condition that effects approximately $2 \%$ of infertile men. ${ }^{1}$ Large scale data set analysis as described here can be helpful for identifying rare or mild CFTR mutations. We observed F508C in 3/182 CBAVD patients, all of whom carried an additional CFTR mutation. One of the additional mutations represents a mild allele (L206W, Table 1). ${ }^{2} \mathrm{We}$ noted F508C in 3/850 CF carrier screens, and none of these individuals carried F508C in conjunction with another disease causing mutation. F508C was observed in 23/5,938 individuals submitted for diagnostic testing on suspicion of CF.
The NCBI SNP database does not provide information regarding the frequency of the $\mathrm{F} 508 \mathrm{C}$ allele, ${ }^{14}$ although a handful of smaller studies have been performed to examine F508C among CBAVD patients, CF subjects or healthy controls. $22,24-26$ The F508C mutation was found to be extremely rare in healthy individuals $(0-0.08 \%) .^{22,24-26}$ However, higher frequencies in CF subjects $(0.2-0.7 \%),{ }^{24,25}$ and among those with CBAVD (1-1.4\%) were noted.22,24 The present findings represent the largest study to date of the F508C mutation, and provide additional evidence for increased frequency of this allele among subjects with CBAVD. The extremely low frequency of F508C observed among normal individuals, during CF carrier screening, and among subjects genotyped for suspicion of CF together with the previously demonstrated mild functional defects ${ }^{24}$ suggest that F508C is in fact a mild pathogenic mutation that can result in CBAVD, and should be viewed in this light as part of genetic screening for either CBAVD or atypical CF.

\section{ACKNOWLEDGMENTS}

We thank the staff of Ambry Genetics for expert technical help in processing 6970 specimens.

\section{References}

1. Grangeia A, Niel F, Carvalho F, et al. Characterization of cystic fibrosis conductance transmembrane regulator gene mutations and IVS8 poly(T) variants in Portuguese patients with congenital absence of the vas deferens. Hum Reprod 2004;19:25022508.

2. Cystic Fibrosis Mutation Database Toronto: The Hospital for Sick Children, Genetics and Genomics Biology, 1989. Available at: http://www.genet.sickkids.on.ca/cftr/ app. Accessed March, 2008.

3. Rowe SM, Miller S, Sorscher EJ. Cystic fibrosis. N Engl J Med 2005;352:1992-2001.

4. Taulan M, Girardet A, Guittard C, et al. Large genomic rearrangements in the CFTR gene contribute to CBAVD. BMC Med Genet 2007;8:22.

5. Ratbi I, Legendre M, Niel F, et al. Detection of cystic fibrosis transmembrane conductance regulator (CFTR) gene rearrangements enriches the mutation spectrum in congenital bilateral absence of the vas deferens and impacts on genetic counseling. Hum Reprod 2007;22:1285-1291.

6. Kerem E. Atypical CF and CF related diseases. Paediatr Respir Rev 2006;7 (suppl 1):S144-S146.

7. Danziger KL, Black LD, Keiles SB, Kammesheidt A, Turek PJ. Improved detection of cystic fibrosis mutations in infertility patients with DNA sequence analysis. Hum Reprod 2004;19:540-546.

8. Cuppens H, Cassiman JJ. CFTR mutations and polymorphisms in male infertility. Int J Androl 2004;27:251-256.

9. Chillon M, Casals T, Mercier B, et al. Mutations in the cystic fibrosis gene in patients with congenital absence of the vas deferens. N Engl J Med 1995;332:1475-1480.

10. Kopito RR. Biosynthesis and degradation of CFTR. Physiol Rev 1999;79:S167-S173.

11. Du K, Sharma M, Lukacs GL. The DeltaF508 cystic fibrosis mutation impairs domain-domain interactions and arrests post-translational folding of CFTR. Nat Struct Mol Biol 2005;12:17-25.

12. Cui L, Aleksandrov L, Hou YX, et al. The role of cystic fibrosis transmembrane conductance regulator phenylalanine 508 side chain in ion channel gating. J Physiol 2006;572:347-358.

13. Kobayashi K, Knowles MR, Boucher RC, O’Brien WE, Beaudet AL. Benign missense variations in the cystic fibrosis gene. Am J Hum Genet 1990;47:611-615.

14. NCBI Single Nucleotide Polymorphisms Database National Center for Biotechnology Information, 1998. Available at: http://www.ncbi.nlm.nih.gov/projects/SNP/. Accessed June, 2008.

15. Vassar College, 1998. Available at: http://faculty.vassar.edu/lowry/VassarStats.html. Accessed November, 2007.

16. Macek M Jr, Ladanyi L, Burger J, Reis A. Missense variations in the cystic fibrosis gene: heteroduplex formation in the F508C mutation. Am J Hum Genet 1992;51:1173-1174.

17. Desgeorges M, Kjellberg P, Demaille J, Claustres M. A healthy male with compound and double heterozygosities for delta F508, F508C, and M47OV in exon 10 of the cystic fibrosis gene. Am J Hum Genet 1994;54:384-385. 


\section{Havasi et al.}

18. Dufourcq R, Vuillaumier S, Pascaud O, et al. Compound heterozygosity for delta F508 and F508C: a cautionary note on the molecular diagnosis of cystic fibrosis. Prenat Diagn 1994;14:1176-1177.

19. Warren WS, Hamosh A, Egan M, Rosenstein BJ. False-positive results of genetic testing in cystic fibrosis. J Pediatr 1997;130:658-660.

20. Kerem BS, Zielenski J, Markiewicz D, et al. Identification of mutations in regions corresponding to the two putative nucleotide (ATP)-binding folds of the cystic fibrosis gene. Proc Natl Acad Sci USA 1990;87:8447-8451.

21. Kalin N, Dork T, Tummler B. A cystic fibrosis allele encoding missense mutations in both nucleotide binding folds of the cystic fibrosis transmembrane conductance regulator. Hum Mutat 1992;1:204-210.

22. Traystman MD, Schulte N, Colombo JL, et al. Mutation analysis and haplotype correlation for 139 cystic fibrosis patients from the Nebraska Regional Cystic Fibrosis Center. Hum Mutat 1993;2:7-15.
23. Meschede D, Eigel A, Horst J, Nieschlag E. Compound heterozygosity for the delta F508 and F508C cystic fibrosis transmembrane conductance regulator (CFTR) mutations in a patient with congenital bilateral aplasia of the vas deferens. Am J Hum Genet 1993;53:292-293.

24. Dork T, Dworniczak B, Aulehla-Scholz C, Wieczorek D, et al. Distinct spectrum of CFTR gene mutations in congenital absence of vas deferens. Hum Genet 1997;100: 365-377.

25. Pallares-Ruiz N, Carles S, Des Georges M, et al. Complete mutational screening of the cystic fibrosis transmembrane conductance regulator gene: cystic fibrosis mutations are not involved in healthy men with reduced sperm quality. Hum Reprod 1999;14:3035-3040.

26. Pompei F, Ciminelli BM, Bombieri C, et al. Haplotype block structure study of the CFTR gene. Most variants are associated with the M470 allele in several European populations. Eur J Hum Genet 2006;14:85-93. 